\title{
Colección de Mamíferos, Museo de Historia Natural C.J. Marinkelle, Universidad de los Andes (ANDES-M), Colombia
}

\author{
Sebastián García-R ${ }^{1 *}$ (iD \\ 1 Departamento de Ciencias Biológicas, Universidad de los Andes, Museo de Historia Natural C.J. Marinkelle. Carrera 1 \# 18a- \\ 12, 111711, Bogotá D.C., Colombia. \\ *Correspondencia: s.garciar@uniandes.edu.co
}

\section{Resumen}

La Colección de Mamíferos del Museo de Historia Natural C.J. Marinkelle (ANDES-M), cuenta con 2466 especímenes, catalogados desde la década de 1960, en los cuales están representados 11 órdenes, 29 familias, 82 géneros y 132 especies. Su representatividad geográfica es de seis países, incluyendo Colombia. Los especímenes proceden de 27 departamentos, principalmente del Meta y Santander.

Palabras clave: Chiroptera, especímenes, Mastozoología, Uniandes.

\section{Abstract}

The Mammal Collection of the "C.J. Marinkelle" Natural History Museum (ANDES-M), has 2466 cataloged specimens, collected since the 1960s, which are represented by 11 orders, 29 families, 82 genera and 132 species. The geographic coverture covers six countries, including Colombia. The specimens come from 27 Colombian departments, mainly Meta and Santander.

Key words: Chiroptera, Mammalogy, specimens, Uniandes.

La Colección de Mamíferos del Museo de Historia Natural C.J. Marinkelle (ANDES-M) (Figura 1), está ubicada en Bogotá D.C. El museo que alberga la colección se creó a principios de la década de 1960 durante la estancia de James Ray Tamsitt y Darío Valdivieso en la Universidad de los Andes, para albergar el material mastozoológico proveniente de estudios zoonóticos en el país (Mantilla-Meluk 2010). En sus publicaciones sobre murciélagos de Colombia, Tamsitt y Valdivieso hacen mención del naciente museo (Valdivieso \& Tamsitt 1962; Tamsitt \& Valdivieso 1962a; Tamsitt \& Valdivieso 1963a,b; Valdivieso 1964; Tamsitt et al. 1964; Tamsitt \& Valdivieso 1964a; Valdivieso \& Tamsitt 1965). En esta etapa inicial resulta interesante resaltar la participación de Jorge Hernández Camacho en la identificación de especímenes (Valdivieso \& Tamsitt 1962; Tamsitt \& Valdivieso 1963a,b; Tamsitt \& Valdivieso 1964a). Se ha considerado que las publicaciones de Tamsitt y Valdivieso (Valdivieso \& Tamsitt 1962; Tamsitt \& Valdivieso 1962a) dieron inicio formalmente a los estudios sobre murciélagos en Colombia (Mantilla-Meluk et al. 2014). Posteriormente, tras la salida de Tamsitt en 1963, Cornelis Johannes Marinkelle se vincula 
al Departamento de Ciencias Biológicas quedando a cargo de la línea de Mastozoología con estudios enfocados principalmente en parasitología (Guhl 2012). Esto llevó al crecimiento de las colecciones del museo a partir del material biológico obtenido en sus muestreos en la Orinoquía, Amazonía y piedemonte oriental de la cordillera Oriental de los Andes (Mantilla-Meluk et al. 2014). Adicionalmente, Alberto Cadena, durante su estancia como estudiante de pregrado ( 1964-1965) y posteriormente como profesor en la Universidad de los Andes (1967-1969 y 1972-1975), trabajó con C. J. Marinkelle aportando registros importantes para la mastofauna de Colombia (Sociedad Colombiana de Mastozoología 2010, Mantilla-Meluk 2010, Mantilla-Meluk et al. 2014).

El actual museo fue inaugurado en septiembre de 2006, reuniendo el material biológico de diferentes colecciones que se encontraba disperso en laboratorios de docencia e investigación. El 20 de abril de 2020, el Departamento de Ciencias Biológicas le concede el nombre de C.J. Marinkelle como reconocimiento a uno de sus profesores eméritos.

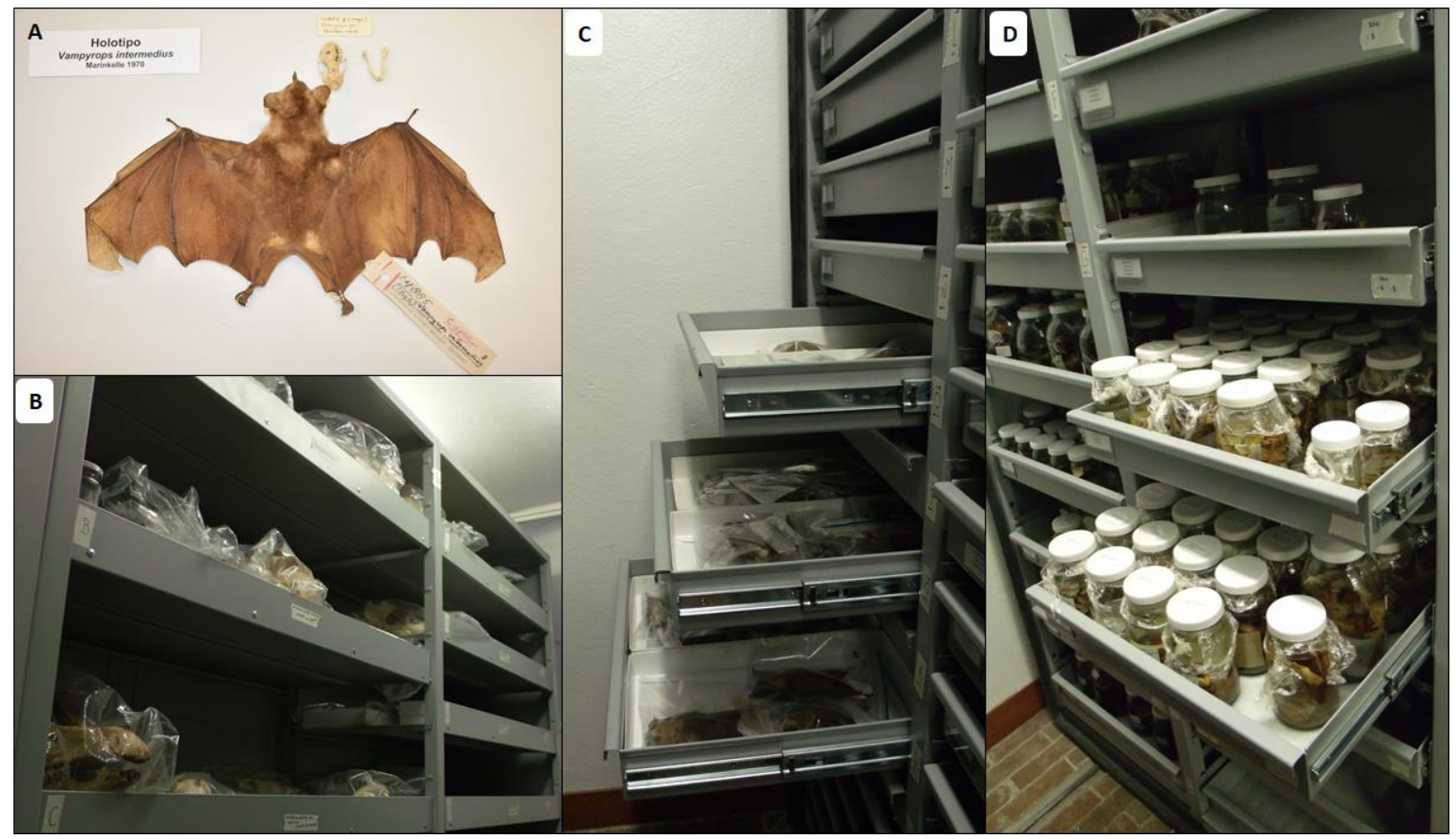

FIGURA 1. Detalles de la Colección de Mamíferos (ANDES-M). A) Holotipo de Vampyrops intermedius Marinkelle 1970; B) Sección en seco, mamíferos medianos; C) Sección en seco, mamíferos pequeños; D) Sección en líquido.

En la actualidad, la Colección de Mamíferos alberga 2466 especímenes catalogados (conteo a 14 abril de 2020) pertenecientes a 11 órdenes, 29 familias, 82 géneros (1 exótico: Rattus) y 138 especies (1 exótica: Rattus rattus) (Tabla 1). El orden mejor representado es Chiroptera (2340 especímenes) con 105 especies. Los especímenes provienen principalmente de Colombia (2346 especímenes), Argentina (4), Bolivia (1), Ecuador (20), Estados Unidos (27) y Perú (2). 66 especímenes no cuentan con información de la localidad. La colección cuenta con una representatividad para 27 departamentos de Colombia, y aproximadamente 80 localidades nacionales. Los especímenes proceden de los departamentos de Amazonas (178), Antioquia (4), Arauca (1), Atlántico (3), Bolívar (237), Boyacá (6), Caldas (2), Caquetá (25), Casanare (4), Cauca (62), Cesar (4 ), Chocó (21), Córdoba (1), Cundinamarca (43), Guainía (1), Guaviare (17 ), Huila (1), La Guajira (37), Magdalena (9), Meta (791), Putumayo (31), 
Risaralda (9), Santander (654), Tolima (119), Valle del Cauca (35), Vaupés (38) y Vichada (3); y 10 sin información de localidad nacional.

Un total de 2464 especímenes están identificados a nivel de orden, 2445 a familia, 1843 a género y 1654 a especie. En el momento la colección cuenta con 118 especímenes en proceso de ingreso a catálogo (Chiroptera 67,8\%; Didelphimorphia 4,2\% y Rodentia 28\%) provenientes de Chocó (Capurganá), Cundinamarca (La Mesa) y Casanare (Maní y Tauramena). Estas colectas corresponden a salidas de campo del curso "Colecciones biológicas" y a proyectos del Centro de Investigaciones en Microbiología y Parasitología Tropical CIMPAT de la Universidad de los Andes.

TABLA 1. Órdenes, familias, géneros y especies de mamíferos depositados en la colección de Mamíferos ANDES-M.

\begin{tabular}{lcccc}
\hline \multicolumn{1}{c}{ Orden } & Familias & Géneros & Especies & Número de especímenes \\
\hline Didelphimorphia & 1 & 4 & 4 & 16 \\
Paucituberculata & - & - & - & - \\
Cingulata & - & - & - & - \\
Pilosa & 2 & 2 & 2 & 2 \\
Sirenia & 1 & 1 & 1 & 2 \\
Eulipotyphla & - & - & - & - \\
Chiroptera & 8 & 51 & 105 & 2340 \\
Carnivora & 2 & 3 & 3 & 3 \\
Perissodactyla & 1 & 1 & 1 & 1 \\
Artiodactyla & 2 & 3 & 3 & 11 \\
Cetacea & 1 & 1 & 2 & 5 \\
Primates & 2 & 4 & 4 & 11 \\
Rodentia & 8 & 11 & 12 & 72 \\
Lagomorpha & 1 & 1 & 1 & 1 \\
Total & 29 & 82 & 138 & 2464 \\
\hline
\end{tabular}

Especímenes de la Colección de Mamíferos ANDES-M, han sido empleados en al menos 24 trabajos de investigación (Valdivieso \& Tamsitt 1962; Tamsitt \& Valdivieso 1962a; Tamsitt \& Valdivieso 1963a,b; Valdivieso 1964; Tamsitt et al. 1964; Tamsitt \& Valdivieso 1964a; Valdivieso \& Tamsitt 1965; Marinkelle 1970; Marinkelle \& Cadena 1971; Marinkelle \& Cadena 1972; Tamsitt et al. 1986; Tamsitt \& Valdivieso 1986; Alberico et al. 2000; Cuartas-Calle \& Muñoz-Arango 2003; Ramírez-Chaves 2008; Ramírez-Chaves \& Noguera-Urbano 2010; Ramírez-Chaves \& Pérez 2010; Ramírez-Chaves 2011; Ramírez-Chaves et al. 2013; PérezTorres et al. 2015; Bautista-Plazas et al. 2017; Herrera-Alvarez et al. 2018; Esquivel et al. 2020). Existen publicaciones relacionadas con biología reproductiva y parasitología, que mencionan a la Universidad de los Andes, pero no son claros en cuanto al uso de material depositado en la colección de mamíferos (Tamsitt \& Valdivieso 1962b; Tamsitt \& Valdivieso 1963c; Tamsitt \& Valdivieso 1964b; Grose \& Tamsitt 1965; Tamsitt \& Valdivieso 1965; Marinkelle \& Grose 1981). El número de citaciones de los artículos que incluyen especímenes de la colección de Mamíferos ANDES-M se encuentra disponible en el siguiente enlace: https:// bit.ly/3cuch4B

La colección alberga el holotipo de Vampyrops intermedius Marinkelle 1970, que actualmente se considera sinónimo reciente de Platyrrhinus infuscus Peters 1880 (Gardner 


eniente de

\& Carter 1972). El ejemplar, piel con número 14885, es una hembra proveniente de la “Mina de Upín" cerca al municipio de Restrepo, Meta (4.3, -73.5667). En la descripción se asignaron 17 paratipos, depositados en el museo de la Universidad de los Andes y en el Instituto de Ciencias Naturales de la Universidad Nacional de Colombia (Marinkelle 1970). De estos, actualmente sólo se tiene información de cinco especímenes depositados en el museo de la Universidad de los Andes $(13310,13445,13451,13452,13453)$ y uno en el Instituto de Ciencias Naturales (14884, ICN 18472; Ramírez-Chaves 2011).

Entre los principales recolectores en la Colección de Mamíferos ANDES-M, se encuentran C. J. Marinkelle, J. R. Tamsitt y D. Valdivieso durante sus primeros años. Recientemente se encontraron especímenes que datan de 1964 y 1968, con etiquetas del Museum of Texas Tech University, y números de colector de Robert Baker, R.D.I Kewberry, Kenneth George Matocha, Tony Ray Mollhagen y Robert W. Wiley. La información en etiquetas incluye las identificaciones de Corynorhinus townsendii, Lasionycteris noctivagans, Lasiurus borealis, Lasiurus cinereus, Nycticeius humeralis y Pipistrellus hesperus, de los estados de Texas, Colorado y New Mexico. Esto da cuenta de las relaciones de colaboración entre los investigadores de la época con mastozoólogos norteamericanos.

\section{AGRADECIMIENTOS}

A Emilio Realpe, director del Museo "C.J. Marinkelle", Yiselle Cano y Alberto Farfán por su acompañamiento en el acceso a bases de datos y en el ingreso de material biológico. A los estudiantes de pregrado de la Universidad de los Andes que han dedicado una parte de su tiempo a actividades de organización y preservación de especímenes, y a Sergio Solari por su asesoría en tareas de preservación y curaduría del material biológico.

\section{REFERENCIAS}

Alberico M, Cadena A, Camacho JH, Muñoz-Saba Y. 2000. Mamíferos (Synapsida: Theria) de Colombia. Biota colombiana 1:43-75.

Bautista-Plazas S, Rodríguez-Bolaños A, Castellanos R, Stevenson PR. 2017. First inventory of small non-flying mammals of the Cueva de los Guacharos National Natural Park (Huila Colombia). Mammalogy Notes. 4:18-21.

Cuartas-Calle CA, Muñoz-Arango J. 2003. Lista de los Mamíferos (Mammalia: Theria) del departamento de Antioquia Colombia. Biota Colombiana. 4:65-78.

Esquivel DA, Penagos AP, García-R S, Bennett D. 2020. New records of Pygmy Round-eared Bat Lophostoma brasiliense Peters 1867 (Chiroptera Phyllostomidae) and updated distribution in Colombia. Check List. 16:277-285.

Gardner AL, Carter DC. 1972. A Review of the Peruvian Species of Vampyrops (Chiroptera: Phyllostomatidae). Journal of Mammalogy. 53:72-82. https:// doi.org/10.2307/1378828

Grose E, Tamsitt JR. 1965. Paracoccidioides brasiliensis recovered from the intestinal tract of three bats (Artibeus lituratus) in Colombia SA. Sabouraudia. 4:124-125. https:/ / doi.org/10.1080/00362176685190281

Guhl F. 2012. Cornelis Johannes Marinkelle biólogo médico Ph. D. Infectio. 16:6-7.

Herrera-Alvarez S, Karlsson E, Ryder OA, Lindblad-Toh K, Crawford AJ. 2018. How to make a rodent giant: Genomic basis and tradeoffs of gigantism in the capybara the world's largest rodent. bioRxiv. 424606 . 
Mantilla-Meluk H. 2010. Sobre la conformación de la Sociedad Colombiana de Mastozoología (SCMas). Mastozoología Neotropical. 17:257-261.

Mantilla-Meluk H, Cadena-G A, Jiménez-Ortega AM. 2014. Historia de la mastozoología en Colombia: Pasado presente y perspectivas. En: Ortega J, Martínez JL, y Tirira DG, editores. Historia de la mastozoología en Latinoamérica las Guayanas y el Caribe. Editorial Murciélago Blanco y Asociación Ecuatoriana de Mastozoología Quito y México DF. p 153-174.

Marinkelle CJ. 1970. Vampyrops intermedius sp. n. from Colombia (Chiroptera Phyllostomatidae). Revista Brasilera de Biología. 30:49-53.

Marinkelle CJ, Cadena A. 1971. Remarks on Sturnira tildae in Colombia. Journal of Mammalogy. 52: 235-237. https:// doi.org/10.2307/1378460

Marinkelle CJ, Cadena A. 1972. Notes on bats new to the fauna of Colombia. Mammalia. 36:50-58.

Marinkelle CJ, Grose ES. 1981. A list of ectoparasites of Colombian bats. Revista de Biología Tropical. 29:11-20.

Pérez-Torres J, Estrada-Villegas S, Martínez-Medina D, Ríos-Blanco M, Peñuela-Salgado M, MartínezLuque L. 2015. Macaregua: the cave with the highest bat richness in Colombia. Check List. 11: 1-6. https:// doi.org/10.15560/11.2.1616

Ramírez-Chaves HE. 2008. Revisión taxonómica del género de murciélagos Eptesicus rafinesque, 1820 (Mammalia: Chiroptera: Vespertilionidae) para Colombia. Tesis de Maestría en CienciasBiología Universidad Nacional de Colombia.

Ramírez-Chaves HE, Noguera-Urbano EA. 2010. Lista preliminar de los mamíferos (Mammalia: Theria) del departamento de Nariño Colombia. Biota Colombiana. 11:117-140.

Ramírez-Chaves HE, Pérez WA. 2010. Mamíferos (Mammalia: Theria) del departamento del Cauca Colombia. Biota Colombiana. 11:141-171.

Ramírez-Chaves HE. 2011. Especímenes tipo de mamíferos en la colección del Instituto de Ciencias Naturales Universidad Nacional de Colombia. Acta Biológica Colombiana.16:281-292.

Ramírez-Chaves HE, Noguera-Urbano EA, Rodríguez-Posada ME. 2013. Mamíferos (Mammalia) del departamento de Putumayo Colombia. Revista de la Academia Colombiana de Ciencias Exactas Físicas y Naturales. 37:263-286.

Sociedad Colombiana de Mastozoología. 2010. Comunicado Oficial No. 002: Elección Fiscal SCMas. Recuperado de https:// bit.ly/3cRCIGx

Tamsitt JR, Valdivieso D. 1962a. Desmodus rotundus rotundus from a high altitude in southern Colombia. Journal of Mammalogy. 43:106-107. https:// doi.org/10.2307/1376890

Tamsitt JR, Valdivieso D. 1962b. Le cycle oestral du rat sigmodon bogotensis de Colombie. Mammalia. 26:161-166. doi: https:// doi.org/10.1515/mamm.1962.26.2.161

Tamsitt JR, Valdivieso D. 1963a. Records and observations on Colombian bats. Journal of Mammalogy. 44:168-180. https://doi.org/10.2307/1377449

Tamsitt JR, Valdivieso D. 1963b. Notes on bats from Leticia Amazonas Colombia. Journal of Mammalogy. 44:263. https:// doi.org/10.2307/1377458

Tamsitt JR, Valdivieso D. 1963c. Reproductive cycle of the big fruit-eating bat Artibeus lituratus Olfers. Nature. 198:104.

Tamsitt JR, Valdivieso D, Hernández-Camacho J. 1964. Bats of the Bogota savanna Colombia with notes on altitudinal distribution of neotropical bats. Revista de Biología Tropical. 12:107-115.

Tamsitt JR, Valdivieso D. 1964a. Notes on some mammals from Colombia. Journal of Mammalogy. 45: 324-327. https:// doi.org/10.2307/1377019 
Tamsitt JR, Valdivieso D. 1964b. Informations sur la reproduction des cheiroptères phyllostomides de Colombie. Mammalia. 28:397-402. https:// doi.org/10.1515/mamm.1964.28.3.397

Tamsitt JR, Valdivieso D. 1965. The male reproductive cycle of the bat Artibeus lituratus. The American Midland Naturalist. 73:150-160. https:/ / doi.org/10.2307/2423327

Tamsitt JR, Cadena A, Villarraga E. 1986. Records of bats (Sturnira magna and Sturnira aratathomasi) from Colombia. Journal of Mammalogy. 67:754-757. https://doi.org/10.2307/1381141

Tamsitt JR, Valdivieso D. 1986. Variación morfométrica en el murciélago Sturnira magna (Chiroptera: Phyllostomidae). Caldasia. 15:743-760.

Valdivieso D, Tamsitt JR. 1962. First records of the Pale Spear-Nosed Bat in Colombia. Journal of Mammalogy. 43:422-423. https://doi.org/10.2307/1376961

Valdivieso D. 1964. La fauna quiróptera del departamento de Cundinamarca Colombia. Revista de Biología Tropical. 12:19-45.

Valdivieso D, Tamsitt JR. 1965. The histology of the chest gland of the Pale Spear-Nosed Bat. Journal of Mammalogy. 45:536-539. https:// doi.org/10.2307/1377325

Editor: Héctor E. Ramírez-Chaves

Recibido: 2020-04-19

Revisado: 2020-04-26

Aceptado: 2020-04-27

Publicado: 2020-06-01 